\title{
Rendimiento académico universitario en países latino americanos bajo la neurocomputación biológica
}

\section{University academic performance in latin american countries under the biological neurocomputing}

\author{
JIMÉNEZ-GALÁN, Joel Luis†*, FALCONE-TREVIÑO, Giuseppe Francisco, TINAJERO- \\ MALLOZZI, Zaida Leticia y SERNA-HINOJOSA, José Antonio
}

Universidad Autónoma de Tamaulipas

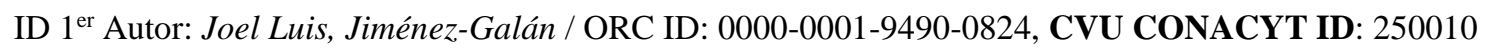

ID $1^{\mathrm{er}}$ Coautor: Giuseppe Francisco, Falcone-Treviño / ORC: 0000-0003-0459-9834, CVU CONACYT ID 240812

ID $2^{\text {do }}$ Coautor: Zaida Leticia, Tinajero-Mallozzi / ORC ID: 0000-0003-1397-4632, CVU CONACYT ID 616868

ID $3^{\text {er }}$ Coautor: José Antonio, Serna-Hinojosa / ORC ID: 0000-0003-0099-9055, CVU CONACYT ID 637084

DOI: $10.35429 / J U M .2019 .10 .3 .20 .34$

Recibido 19 de Octubre, 2019; Aceptado 03 de Diciembre, 2019

Resumen

Primero, que se pueda identificar si el rendimiento académico y habilidades psicosociales, bajo la identidad latinoamericana en el concepto universitario en argentinos, peruanos, costarricenses y mexicanos, se incremente para sus alumnos, considerando la mejora en el desarrollo del estudio académico; segundo, analizar que el aprovechamiento académico al considerar la neurocomputación biológica permita identificar el grado de ansiedad en los estudiantes; tercero, medir los distintos indicadores entre las habilidades sociales y los promedios académicos entre los alumnos. Mediante su diseño observacional multicéntrico enfocado a la neurocomputación biológica, se utilizó un instrumento de medición en la escala MEDMAR, con una muestra finita del universo estudiantil del 1500, con diferentes ambientes escolares en las universidades de Universidad Nacional de Rosario (Argentina), Universidad Nacional Mayor de San Marcos de Lima (Perú), Universidad de Costa Rica (Costa Rica), Universidad de Guadalajara (México), Universidad de Aguascalientes (México) y Universidad Autónoma de Tamaulipas (México) en el 2018. Con indicadores sobre lo afectivo/emocional. El rendimiento académico y el comportamiento de los alumnos, se detectó que un $20 \%$ de alumnos con falta de motivación para su aprendizaje. Se definieron situaciones de carácter neurobiológico computacional con interacción hacia los programas educativos, al manejar el rendimiento académico óptimo y la constante carencia de interés por el nuevo conocimiento por adquirir con datos de consistencia interna, fiabilidad test-retest y validez concurrente.

\section{Aprendizaje, Factores de riesgo, Rendimiento} académico

\begin{abstract}
First, that it can be identified if academic performance and psychosocial skills, under the Latin American identity in the university concept in Argentines, Peruvians, Costa Ricans and Mexicans, increase for their students, considering the improvement in the development of the academic study; second, to analyze that academic achievement when considering biological neurocomputing allows to identify the degree of anxiety in students; third, measure the different indicators between social skills and academic averages among students. Through its multicenter observational design focused on biological neurocomputing, a measuring instrument was used on the MEDMAR scale, with a finite sample of the student universe of 1500, with different school environments in the universities of the National University of Rosario (Argentina), National University Mayor of San Marcos de Lima (Peru), University of Costa Rica (Costa Rica), University of Guadalajara (Mexico), University of Aguascalientes (Mexico) and Autonomous University of Tamaulipas (Mexico) in 2018. With indicators on the affective / emotional. The academic performance and behavior of the students, it was detected that $20 \%$ of students with lack of motivation for their learning. Situations of computational neurobiological character were defined with interaction towards educational programs, when managing the optimal academic performance and the constant lack of interest in the new knowledge to acquire with data of internal consistency, test-retest reliability and concurrent validity.
\end{abstract}

Learning, Risk factors, Academic performance

Citación: JIMÉNEZ-GALÁN, Joel Luis, FALCONE-TREVIÑO, Giuseppe Francisco, TINAJERO-MALLOZZI, Zaida Leticia y SERNA-HINOJOSA, José Antonio. Rendimiento académico universitario en países latino americanos bajo la neurocomputación biológica. Revista de Gestión Universitaria. 2019 3-10: 20-34

\footnotetext{
* Correspondencia del Autor (correo electrónico: jjimenez@ docentes.uat.edu.mx)

$\dagger$ Investigador contribuyendo como primer autor. 


\section{Introducción}

Se pudo clasificar a la ansiedad como un estado emocional y actitud de conducta que en la mayoría los estudiantes a nivel superior mostraron determinada química y biología neuronal considerada como muy incapacitante, grave, moderada, leve o ausente a ciertos niveles, sobre todo cuando hay retos que mostrar sobre los sentimientos y emociones, al realizar ciertas actividades como: la exposición de un trabajo final en el aula o frente a los compañeros y docentes, así también al presentar algún tipo de test, ya sea escrito u oral, o simplemente al estar esperando algún resultado sobre una asignatura en su calificación.

La región latinoamericana y caribeña muestra en la actualidad un gran déficit en el logro de una confluencia que derive en cursos de acción cooperativos acerca de los grandes temas de la agenda global y regional. (Caetano, 2015).

Partiendo de la idea que vivir situaciones difíciles o complicadas son incomodidades, por ejemplo, al ser recibido por un prefecto a la entrada de la escuela y que te impida el ingreso a la institución o por algún profesor quien no te dio la oportunidad de ingresar al aula y en ese momento se convierta en un sentimiento de violencia, autoritarismo, paternalismo o estado coercitivo.

También como menciona (Jiménez. 2011). "Ambiente escolar hostil, tensión, violento, bullying, acoso de algún docente o compañero de clase, que pueda ocasionar desmotivación, falta de entusiasmo o sienta temores, tenga insomnio, dificultad para concentrarse, mala memoria, tristeza, dolores musculares, síntomas cardiovasculares como taquicardia, constricción en el pecho, disnea, dispepsia, sensación de estómago lleno, etc.".

Considerando las variables manejadas en el rendimiento académico se puede decir "El aula de clase es una microsociedad cuya finalidad esencial consiste en elaborar conocimiento compartido y en un marco de interacción" (Roselli, 2011).
En Argentina por ejemplo se menciona que composición étnica de la población de la Argentina es, en orden cronológico, el resultado de la mestización de la población indígenanativa precolombina con una relativamente pequeña población de colonizadores europeos ibéricos y con una importante población de origen africano-subsahariano inmigrada forzosamente y esclavizada (la cual dio origen a la población afroargentina), todo en la época colonial.

A esta población, que formó la totalidad de la población argentina hasta aproximadamente 1860 , se le sumó la gente proveniente de la gran ola de inmigración europea, mayoritariamente italiana y española, Ley Nacional de Educación (2007). Ley $\mathrm{N}^{\circ}$ 26.206. "CAPITULO I. Derechos, Artículo XII. Toda persona tiene el derecho de participar en la vida cultural de la comunidad, gozar de las artes y disfrutar de los beneficios que resulten de los progresos intelectuales y especialmente de los descubrimientos científicos; articulo III, La educación que imparta el Estado tenderá a desarrollar armónicamente, todas las facultades del ser humano y fomentará en él, a la vez, el amor a la Patria, el respeto a los derechos humanos y la conciencia de la solidaridad internacional, en la independencia y en la justicia”. la pregunta que surge es: ¿la escuela debe adaptarse totalmente a los avatares de los estudiantes? La respuesta no es simple, porque, a la tentación de exigirle a la escuela una total flexibilización normativa para garantizar la retención, se opone el convencimiento de la necesidad de un mínimo de reglas básicas para el funcionamiento de toda institución en general $\mathrm{y}$, en particular, de una experiencia escolar con algún grado de intensidad" (Kessler, 2004).

En otro país como Costa Rica, la genética de los costarricenses del siglo XXI está compuesta principalmente por cuatro grupos étnicos, o mejor llamados ancestrías, pues este término refiere más a un sentido histórico. Así lo dieron a conocer recientemente tres investigadores del Centro de Investigación en Biología Celular y Molecular (CIBCM) de la Universidad de Costa Rica (UCR), en su estudio sobre la composición genética de los costarricenses.

El estudio realizado por (Campos, R., Barrantes, R. \& Raventós, H. 2016), analizó una muestra de 160 individuos para conocer de dónde procedían sus genes.

JIMÉNEZ-GALÁN, Joel Luis, FALCONE-TREVIÑO, Giuseppe Francisco, TINAJERO-MALLOZZI, Zaida Leticia y SERNAHINOJOSA, José Antonio. Rendimiento académico universitario en países latino americanos bajo la neurocomputación biológica. Revista de Gestión Universitaria. 2019 
Aunque se trata de una muestra pequeña, Barrantes aseguró que es significativa y precisa, pues es aleatoria e independiente de factores como enfermedades, que se habían considerado en estudios anteriores, lo cual representaba un sesgo.

La forman la genética de los costarricenses. Según el informe, de las analizadas, la europea sigue siendo la predominante, con un $45,6 \%$ de presencia en la muestra. En segundo lugar, se encuentra la amerindia con un $33,5 \%$.

Las ancestrías africana y asiática se presentan en menor medida con un $11,7 \%$ y $9,2 \%$, respectivamente. "El costarricense por sí solo es una mezcla", afirmó la investigadora Campos. Agregó que en este estudio la diferencia fue que no existía ninguna enfermedad o condición específica asociada a la muestra, lo cual significa una fortaleza del trabajo.

A lo largo de la historia nacional, oleadas importantes de migrantes han llegado al país por diferentes razones. Un ejemplo es la comunidad asiática, especialmente china, que inició su migración a mediados del siglo XIX y se ha expandido por todo el territorio nacional desde entonces. Asimismo, ha habido considerables migraciones de europeos y latinoamericanos, como también de africanos durante la colonia. "Nuestra historia se refleja en nuestra genética", aseguró Campos. La investigadora mencionó que uno de los hallazgos más importantes, más allá de los resultados generalizables a nivel nacional, es la diferencia entre individuos.

Existe una "gran variabilidad en la composición genética individual”, dijo. Es decir, entre dos o más individuos analizados, los porcentajes de las cuatro ancestrías varían notablemente.

Por eso en los artículos de la constitución 77 y 78, mencionan:” La educación pública será organizada como un proceso integral correlacionado en sus diversos ciclos, desde la preescolar hasta la universidad y en la educación estatal, incluida la superior, el gasto no será inferior al seis por ciento (6\%) anual del producto interno bruto, de acuerdo con la ley, sin prejuicio de lo establecido en los artículos 84 y 85 de esta constitución.
La identidad del estudiante, a los resultados del progreso académico e intelectuales y una correlación en los ciclos escolarizados. Abarca y Sánchez (2005), encontraron que los desertores tienen un escaso conocimiento sobre las carreras en las que quedan matriculados $\mathrm{h} 32 \mathrm{~g} \mathrm{n}$ ( $\mathrm{h}=$ hombres, $\mathrm{g}=$ género, $\mathrm{n}=$ número) y el no ingreso a la carrera deseada es un factor que estimula el abandono de la universidad. También se reporta que el factor económico no es determinante para la deserción y que los estudiantes abandonan la institución, pero no el sistema universitario, ya que se incorporan a otras opciones.

En México, Por otra parte, según Jiménez (2013), "un proceso de acreditación significa reconocer la competencia técnica de una organización para llevar a cabo ciertas actividades bien definidas de evaluación de la conformidad, a diferencia de una certificación que está más orientada a la evaluación del grado de cumplimiento, la acreditación de un programa académico de nivel superior es el reconocimiento público de su calidad, otorgado por un organismo de acreditación, no gubernamental y oficialmente reconocido por el Consejo para la Acreditación de la Educación Superior, AC (COPAES) o por Comités interinstitucionales para la evaluación de la educación superior (CIEES). según el Artículo 3o. de la constitución mexicana. "Toda persona tiene derecho a recibir educación. El Estado Federación, Estados, Ciudad de México y Municipios, impartirá educación preescolar, primaria, secundaria y media superior. La educación preescolar, primaria y secundaria conforman la educación básica; ésta y la media superior serán obligatorias, considerando los niveles elemental, secundario y medio superior". Donde (PISA, 2015) menciona también se puede observar que, entre estudiantes de 15 años, hay un nivel de satisfacción con la vida de 8,3 en una escala de 0 a 10 (media de la Organización para la Cooperación y Desarrollo Económico (2009): 7.3). Se observa un nivel de motivación de desempeño superior a la media de la Organización para la Cooperación y Desarrollo Económico PISA (2015): el 83\% donde declararon que quieren ser los mejores, hagan lo que hagan (media la Organización para la Cooperación y Desarrollo Económico (2009). $65 \%$ ), y el $96 \%$ están motivados para sacar las notas más altas en todas o en casi todas las asignaturas (media la Organización para la Cooperación y Desarrollo Económico (2009). $83 \%)$.

JIMÉNEZ-GALÁN, Joel Luis, FALCONE-TREVIÑO, Giuseppe Francisco, TINAJERO-MALLOZZI, Zaida Leticia y SERNAHINOJOSA, José Antonio. Rendimiento académico universitario en países latino americanos bajo la neurocomputación biológica. Revista de Gestión Universitaria. 2019 
Con los datos que se tienen, se logró mostrar que en base al estudio mostrado anteriormente que esa Organización para la Cooperación y Desarrollo Económico PISA (2015) y que se verá, si hay o no un avance en Latinoamérica y dentro de una cobertura universal entre todas las universidades participantes, se empleó la escala de Likert como una herramienta de medición que, a diferencia de preguntas dicotómicas con respuesta sí/no, nos permite medir actitudes y conocer el grado de conformidad del encuestado con la finalidad de establecer en los instrumentos una escala como el referente con un sentido común, para cada uno de los ítems considerados de acuerdo con las variables independientes y dependiente utilizadas, en cada una de las encuestas aplicadas a los estudiantes

En México habitan casi 2 millones de afrodescendientes. Los estados con mayor proporción de afrodescendientes son: Guerrero $(6.5 \%)$, Oaxaca (4.9\%) y Veracruz (3.3\%).

Desde principios del siglo pasado, la gran mayoría de los mexicanos han sido clasificados como "mestizos", lo que significa en el uso moderno mexicano que no se identifican plenamente ni con la cultura indígena ni con el patrimonio cultural español, sino que más bien se identifican con rasgos culturales que incorporan elementos de las tradiciones indígenas y españolas.

Los primeros europeos llegaron a territorio mexicano durante la época colonial procedentes en su mayoría de España, principalmente de las regiones de Galicia, Cantabria y el País Vasco.

En México existen personas de Medio oriente y regiones limítrofes tales como árabes, turcos, armenios, iraníes, y judíos y otros del Medio Oriente.

En concreto, la población árabe en México apenas supera el millón, sin embargo, los descendientes de estos han tenido un papel destacado en la vida empresarial, cultural, política y artística de México.

La inmigración de japoneses, coreanos, chinos, filipinos, indios y de otros países del este de Asia, son parte de la composición étnica del país, y todos juntos son casi el $1 \%$ de la población. Anchondo, S. \& de Haro, M. (2016).
En Perú. Bernales Ballesteros Enrique, reconocido escritor y en colaboración con otro escritor de Alberto Otárola (1999), mencionaron que, en la Constitución peruana de 1993, dice. "La cobertura educativa en los niveles básicos y media superior ha tenido resultados favorables, más sin embargo esto no ocurre con la educación superior, ya que en promedio dos terceras partes de los jóvenes en edad de estar estudiando una carrera universitaria, no lo está haciendo", porque analizando las bases de datos del Consejo Nacional de Población (CONAPO, (2011)) y el Consejo Estatal de Población (COESPO) donde se tienen los valores históricos poblacionales, el $45 \%$ de los peruanos son amerindios, el $37 \%$ mestizos, $15 \%$ blancos y el restante $3 \%$ incluye negros, japoneses y chinos (Sandoval, J. \& Rodríguez dos Santos, F. 2013). Así como las proyecciones de crecimiento hasta el año 2030, según la base de datos, por ejemplo, los aspirantes de la Universidad Nacional de San Marcos al querer ingresar a la Universidad Autónoma del Estado de Hidalgo (UAEH) México, se tenía una mejor correlación Pearson con el comportamiento académico y su idea idónea del Consejo Estatal de Población con un rango de jóvenes cuyas edades eran de 15 a 29 años, por medio de la Regresión Lineal, utilizando el software estadístico SPSS ver 10.

Donde se aplicó una ecuación para proyectar a los nuevos aspirantes al 2030, los cuales van a mostrar un crecimiento acumulado de 5.94\% y donde el mismo Consejo Nacional de Población (CONAPO,2012:3) lo proyecta, para el 2050, con una disminución de jóvenes de 18 años de edad que será del $15.7 \%$, si la Universidad Autónoma del Estado de Hidalgo mantiene su crecimiento igual al del periodo anterior del 2011 al 87\% en 2016 (UAEHa:26) que fue de un $6 \%$ anual, se podrá pasar de una cobertura del $34 \%$ en el 2017 a una del $45.9 \%$ y en el 2019 serán aceptados el 100\% de los alumnos del nivel secundaria al nivel medio y así hasta en el 2023".

La Universidad Nacional de San Marcos. Lima. Perú, es una universidad donde los alumnos no pagan, ni inscripción, ni colegiaturas mensuales, es considerada como una institución social, pero al margen de ello también hay condiciones sociales para aceptar a todos los estratos sociales de la sociedad, por ello se precisa que es una universidad totalmente gratuita, frente a otras que son privadas con costo para los alumnos. 
La postura en relación a los docentes nos comenta Ramírez (2009) dice que "antes de avanzar, es necesario definir lo que realmente es un profesor y los nombres que ha recibido para señalarlo, el nombre de maestro, ha tenido a lo largo del tiempo varios sinónimos: profesor, mentor, educador, catedrático, pedagogo incluso enseñante, a las personas que con una preparación específica como el abogado, el doctor, el contador, han hecho de la tarea educativa una profesión y para quienes educar no es una actividad circunstancial, sino trascendente y que constituye el núcleo capital de su vida".

La educación superior comprende: a) carreras universitarias; y b) carreras terciarias en institutos de educación superior (terciarios). La entrada tanto a la universidad como al terciario se realiza una vez terminados los estudios secundarios, Ministerio de Educación de la Nación Argentina, Ferreyra (2009).

La educación secundaria es obligatoria y constituye una unidad pedagógica y organizativa destinada a los/as adolescentes y jóvenes que hayan cumplido con la educación primaria que se divide en dos ciclos. En los últimos ocho años, la inversión educativa ha transitado por un período de aumento histórico. Esto fue posible gracias a una conjunción de factores: un crecimiento del producto mayor al $8 \%$ anual, un fuerte incremento de la presión tributaria (que pasó del $23 \%$ al $33 \%$ en el período), Esta ley estableció una pauta de aumento de inversión en el sector en relación con el Producto Bruto Interno: partiendo de un 4\% en el año 2004, se fijó y superó la meta del 6\% para el 2010. Se estima que cerca del $44 \%$ de los niños de 3 a 5 años no acceden a la educación inicial, y que sólo 31 niños de cada 100 que ingresan a la primaria finalizan el ciclo secundario (Rivas, Vera, \& Bezem, 2010), más aún, cerca de 7\% de la población de entre 13 y 17 años no asiste a la escuela (INFOBASE 2018). Esta exclusión afecta principalmente a los niños y jóvenes de menor nivel socioeconómico: en el quintil de menores ingresos alcanza al $12 \%$ y en el de mayores ingresos sólo al 2\%. En Costa Rica, tales retornos no disminuyeron. El estudio citado sugiere que los retornos educativos en ALC (América Latina y el Caribe) pudieron descender debido a un aumento en la oferta relativa de trabajadores calificados, lo que a su vez pudo ser el resultado de la mejora educativa que experimentaron los países en la década de los noventa.
El objetivo de este trabajo es dar a conocer aspectos sobre el nivel del rendimiento académico que hay en las diferentes carreras universitarias, en los distintos países de América latina, considerando también como afecta la ansiedad para lograr alcanzar un resultado de excelencia académica. Mientras habla sobre el impacto de las últimas leyes del ámbito de la educación aprobadas y el cumplimiento de la norma que fija un mínimo de 180 días de clase, el economista Alieto Guadagni (2015) afirma que: "Y eso que nuestro calendario es el más corto del mundo". El director del (CEA), y miembro de la Academia Nacional de Educación, profundizó: "Son 720 horas, pero si se considera huelgas, feriados puentes y demás en algunas provincias terminan siendo 620". Guadagni explica que los datos en los que se basó su afirmación fueron publicados en el "Compendio mundial de la educación" de la Organización de las Naciones Unidas para la Educación, la Ciencia y la Cultura. Así como también establece Zabalza (2003), indica, que "la competencia profesional es una configuración intelectual que integra en su estructura y funcionamiento una forma de pensar, el manejo de conocimientos formales, $y$ un conjunto de recursos procedimentales $\mathrm{y}$ actitudinales de carácter útil y práctico, en tanto que la profesión la definimos como una práctica social caracterizada por una serie de actividades que se desarrollan con base en un conjunto de conocimientos especializados, capacidades intelectuales y actitudinales que requieren del compromiso personal y la responsabilidad, por parte de quien la ejerce, de actuar tomando en cuenta las repercusiones sociales generadas por su actividad, dado que habrá de constituir una forma de vida".

Sin embargo, recordando a Erazo (2012) indica "que este parámetro es el resultado de múltiples variables de tipo personal y social. En el aspecto personal se tienen características de tipo orgánico, cognitivo, estrategias y hábitos de aprendizaje, motivación, autoconcepto, emoción y conducta; y en el social están las características de tipo familiar, escolar, socioeconómico y cultural". En el artículo que se divide en materiales y métodos, donde se explican las estrategias que se utilizaron para la aplicación de los instrumentos, participación, carreras participantes, facultades y universidades, así como objeto de estudio, criterios de inclusión, captura de los datos y su método representado en figuras y tablas correspondientes. 
También la parte de la discusión referente a los inconvenientes que se tuvieron, como también los logros de la investigación y cuáles serían las líneas nuevas para futuras investigaciones. Se consideraron los resultados y las conclusiones, así como las referencias.

\section{Neurocomputación biológica}

En la búsqueda de estrategias para mejorar los índices de aprobación de estudiantes en las áreas identificadas con mayor reprobación se han realizado análisis predictivos neurobiológicos computacionales. Como la mayoría de los campos de biología, la neurociencia está asumiendo a una "epidomía" de recolección de datos.

Hay proyectos importantes en curso para caracterizar completamente las firmas proteómicas, metabólicas, genómicas y metilómicas para todos los diferentes tipos de neuronas y células gliales en el cerebro humo, con la finalidad de ayudar a la educación fortaleciendo el rendimiento académico. Además, "connec-tomics" planea proporcionar la estructura de red completa de los cerebros, y "synaptomics" tiene como objetivo descubrir todas las moléculas y sus interacciones en las sinapsis.

Este es un buen momento para hacer una pausa y preguntarnos qué esperamos encontrar al final de este inmenso cerebro ómico. Biotic: Area de informática y salud pública. Instituto Carlos III-Proyecto Genoma Humano.

Que comúnmente toman como referencia un elemento de evaluación previa para determinar el estudio de los genes en los alumnos para el examen de selección por carrera. Sin embargo, los estudios publicados se han centrado más en otras áreas de conocimiento como en ciencias de la salud (Gómez et al., 2012) (Huamaní, Gutiérrez y Mezones, 2011), (Vélez y Roa, 2005), (Carrión, 2002), (Tomatis et al., 2016).

México es el país que cuenta con una gran variedad de poblaciones precolombinas que, en muchos casos, han permanecido aisladas, incluso después de la llegada de los europeos hace más de cinco siglos, debido en gran parte a las barreras geográficas del territorio del país, como cadenas montañosas o desiertos.
Fue precisamente ese aislamiento el que, según los investigadores, hizo que los diferentes grupos genéticos de estos países desarrollaran importantes diferencias genéticas y biológicas culturales entre sí, como es el caso de la etnia de los Seri, que habita en la costa noreste del Golfo de California, y los indígenas mayas lacandones que viven en la frontera sur con Guatemala

\section{Materiales y métodos}

En el marco metodológico se observaron las diferentes posturas culturales y sociológicas de los diferentes países latinoamericanos participantes en la investigación, por lo que se considera que hubo una acción libre, voluntaria y dinámica académicamente por participar en cada una de las universidades y facultades, al pedir el consentimiento para la participación en la investigación, donde el docente tuvo que poner especial cuidado para dar las instrucciones a los alumnos participantes, donde muestra estar potencialmente vinculado con él sentir de los alumnos y por tener cierta relación de dependencia o ser consiente del tipo presión que tiene el alumno o que nada tenga que ver con el estudiante en su relación Helsinki de la am (2017).

Identificando, analizando y mediendo, Al momento de aplicar los diferentes instrumentos de rendimiento académico (MEDMAR, 2008) y ansiedad (HAMILTON, 1969), bajo una muestra finita 150 instrumentos en total en las diferentes carreras de licenciaturas, se presentaron varias críticas descritas como: a) cuál es el llenado de los cuestionarios, b) el número de alumnos participantes por encuesta, c) cómo se presentan los formatos, d) el material para el llenado (lápiz, \#2). Aquí es cuando surge el enfoque analítico propuesto por (Lucas, 2001), lo cual menciona "quien analiza los efectos del origen social, tanto en las probabilidades de transición a diferentes niveles". Como en la ubicación biológica de las personas y las elecciones curriculares que hayan adoptado, lo cual señala que un nivel educativo no es universal, los más favorecidos socioeconómicamente utilizan sus ventajas y los recursos para asegurarse el acceso a ese nivel (ventaja cuantitativa); cuando el acceso al nivel se vuelve casi universal, la inequidad de origen no desaparecerá. Es decir, las personas más favorecidas económicamente buscarán ocupar mejores posiciones al interior de un sistema educativo estratificado. 
$\mathrm{Y}$ es que, la situación del conocimiento de las fases del proceso facilita la comprensión de otros procesos utilizados en otras áreas del saber diferentes a la educativa, ya que por ejemplo el proceso empleado en Ciencias Sociales, de acuerdo a Acevedo (2009) este consiste en la generación de una idea de investigación, planteamiento del problema, revisión de la literatura y realización del marco teórico, visualización del alcance del estudio, elaboración de hipótesis y definición de variables, desarrollo del diseño de investigación, definición y selección de la muestra, recolección de datos, análisis de datos y elaboración del reporte de investigación.

La población objeto en la investigación lo constituyen países como: México, Argentina, Perú y Costa Rica, con características propias desde la cultura, genética y biología que computacionalmente se pueden registrar.

Así también el tipo de contratación de los docentes, los diseños curriculares y el programa educativo participante, de acuerdo con su regionalización donde hubo la distribución, así como se analizó una muestra finita del $4.5 \%$ del tamaño del universo 1500 estudiantes de muestreo aleatorio, basado en la fórmula finita: Donde: $\bullet \mathrm{N}=$ Total de la población $\bullet \mathrm{Z} \alpha=1.96$ al cuadrado (si la confiabilidad es del 95\%) $\cdot \mathrm{p}=$ proporción esperada (en este caso $5 \%=0.05) \cdot \mathrm{q}$ $=1-\mathrm{p}($ en este caso $1-0.05=0.95) \cdot \mathrm{d}=$ precisión (en su investigación se usó un 5\%).

De acuerdo con la muestra obtenida utilizando la fórmula para la población finita se obtuvo un tamaño de muestra equivalente a 70 cuestionarios, seleccionada en total, de un universo de 1500 estudiantes, aplicando 14 cuestionarios por aula, en cada facultad, (Medicina, Comercio, Economía, Tecnologías de la Información y Administración) durante los meses de Junio - Diciembre del 2018. Considerando que operativamente si se hubieran seleccionado a más personas, se tendrían dificultades con la implementación de los cuestionarios utilizados, por la necesidad de tener mayor cantidad de encuestadores que se requerirían, así como el tiempo y la movilidad de espacio.

El criterio de inclusión fue que los alumnos estuvieran inscritos en la universidad y en las carreras que se imparten en las facultades.
Donde la cantidad máxima de estudiantes por aula es de 25 , entre las edades de 19 y 21 años, con un género mixto; el llenado de las mismas se llevó a cabo en el salón de clases, a la hora de la impartición de la materia de acuerdo a cada profesor participante en la investigación y su tiempo disponible, el muestreo no es probabilístico por la selección de los alumnos, los criterios fueron de inclusión, a cada alumno se le proporcionó una explicación del llenado de los instrumentos, iniciando con el de rendimiento académico (MEDMAR) que contiene 59 ítems y que están divididos en cinco subgrupos como variables: ambiente escolar, familias, bajo la escala Likert (1 - Totalmente de acuerdo, 2 - De acuerdo, 3 - Ni de acuerdo ni en desacuerdo, 4 - En desacuerdo, 5 - Totalmente en desacuerdo).

Así mismo el instrumento de la ansiedad (HAMILTON), que se divide en dos variables, ansiedad psíquica con siete ítems y ansiedad somática, que también con siete ítems, ambos bajo la escala de Likert (1 - ausente, 2 - leve, 3 Moderado, 4 - Grave, 5 - Muy Grave).

Los alumnos, solo contestaron la parte de la ansiedad somática, se sentaron por filas dejando un mesabanco libre, borrador de goma, todos los alumnos fueron invitados a participar mencionando el motivo de la investigación y donde ellos accedieron con muy buen gusto y ánimo, no hubo exclusión alguna y menos de eliminación, porque a ningún alumno se le obligo para que contestará, más bien fue por invitación a participar en el llenado de los cuestionarios, los alumnos se llevaron aproximadamente 60 minutos en el llenado, donde para el rendimiento académico fue de 40 minutos y para el de ansiedad 20 minutos, se omitió el nombre del alumno por razones confiables y seguridad del encuestado, una vez finalizado el llenado los instrumentos se metieron en un sobre y se selló frente a los alumnos, se creó una matriz para datos que incluyen la codificación de preguntas, campos y posibles respuestas, se recogieron los instrumentos y revisar que estuvieran completamente llenados los ítems, posteriormente se capturaron los datos computacionalmente en el paquete estadístico versión, IBM SPSS, 17.0, para su análisis de la información estadística con el asesoramiento de un especialista en psicología. 
Los instrumentos que se utilizaron en esta investigación fueron publicados bajo el idioma español, están validados y adaptados para el estudio. También se realizaron revisiones a otros ejemplos parecidos con este estudio, con experiencias parecidas sobre diversos autores y su versión original, como son las adaptaciones a otros contextos.

El método empleado fue cuantitativo orientado a conclusiones o manipulación con variables descriptivas cuyo objetivo mencionado, tiene forma síncrona expresada en diseños transversales correlacionales al coeficiente de Pearson, tomando como variable dependiente al rendimiento académico donde se buscaba responder a determinadas preguntas de investigación como:

\section{¿Concluyes todo proyecto que te propones en la escuela?}

En el Gráfico 1 se puede observar los resultados obtenidos en relación a la pregunta, donde la Benemérita Universidad de Guadalajara, México y la Universidad Nacional Mayor de San Marcos de Lima, Perú, los alumnos no tienen muy clara la idea de iniciar y terminar los proyectos en un periodo escolar, seguido por los estudiantes de la Universidad Autónoma de Tamaulipas, México, Universidad Nacional de Rosario, Argentina y Universidad de Aguascalientes, México y posteriormente la Universidad de Costa Rica, Costa Rica que es donde los alumnos si terminan sus proyectos que se propusieron durante las clases.

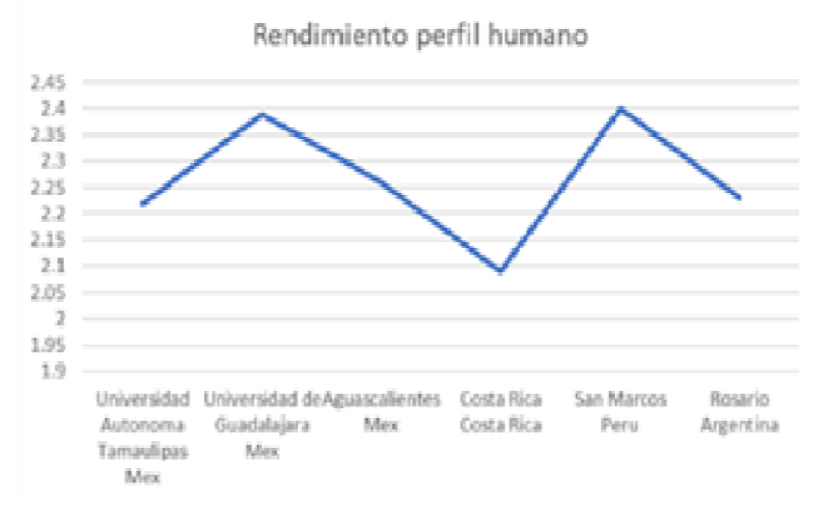

Gráfico 1 Indica los nombres de las universidades participantes para los diferentes países, con relación al rendimiento perfil humano al puntaje correspondiente a la escala de Likert donde 1 - Totalmente de acuerdo, 2 - De acuerdo, 3 - Ni de acuerdo ni en desacuerdo, 4 - En desacuerdo, 5 - Totalmente en desacuerdo.

Fuente: Elaboración Propia

\section{¿Dedicas el tiempo necesario para las labores académica (tareas, estudio)?}

En el Gráfico 2. Se puede observar que hay alumnos que no dedican mucho tiempo al estudio, ni realizan sus tareas los de la Universidad de Aguascalientes obtuvo que sus alumnos tienden a olvidar las tareas o trabajos encargados por el profesor en el salón de clases seguido por la Universidad Autónoma de Tamaulipas y la Universidad Nacional de Rosario, Argentina, posteriormente la Universidad de San Marcos, Lima, Perú, La Universidad de Guadalajara y la Universidad de Costa Rica que donde menos olvidan, realizan sus tareas y cumplen sus trabajos.

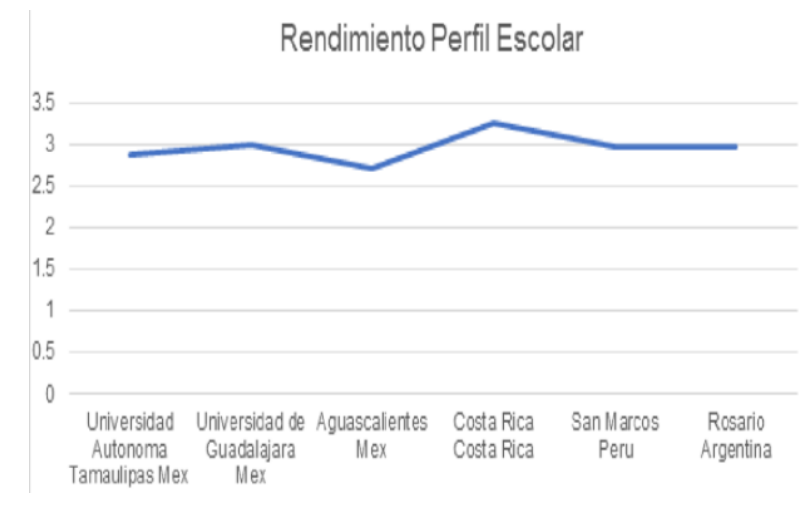

Gráfico 2 Rendimiento Ambiente Escolares. Indica los nombres de las universidades participantes para los diferentes países, con relación al rendimiento ambiente escolar al puntaje correspondiente a la escala de Likert donde 1 - Totalmente de acuerdo, 2 - De acuerdo, 3 - Ni de acuerdo ni en desacuerdo, 4 - En desacuerdo, 5 Totalmente en desacuerdo.

Fuente: Elaboración Propia

\section{Resultados}

Los resultados del análisis de los puntos sobre el rendimiento académico, se logra mediante la participación directa de los países de latino américa involucrados en esta investigación y con la acción decidida y directa de sus universidades interesadas en saber que indicadores y factores son los que están afectando o beneficiando a sus programas académicos en el nivel superior.

Así como las variables independientes que se detallan a continuación en este trabajo y que dan sustento a fortalecer la variable dependiente del rendimiento académico. 


\section{Variable independiente, Rendimiento perfil humano}

En esta variable dentro del rendimiento académico se contemplan 19 ítems donde el primero, ante un problema emocional sé cómo reaccionar; segundo, me considero una persona impulsiva; tercero, considero me conozco a mí mismo; cuarto, me considero un buen amigo; quinto, generalmente tengo una buena condición física; sexto, por lo general me siento fatigado; séptimo, es raro que yo me enferme; octavo, necesito estar limpio en cada momento; noveno, para mi es importante que una persona muestre buenos modales; decimo, soy honesto con las personas; decimo primero, se mantener la calma en situaciones estresantes; décimo segundo, usualmente cumplo las promesas que hago; décimo tercero, concluyo todo proyecto que me propongo; décimo cuarto, me gusta asumir el papel de líder; décimo quinto, me gusta sugerir propuestas novedosas; décimo sexto, por lo general mis relaciones con mis superiores es buena; décimo séptimo, tengo una visión definida de lo que haré cuando termine mi carrera; décimo octavo, tengo una visión clara de lo que estaré haciendo dentro de diez años; décimo noveno, estoy haciendo todo lo que está en mis manos para terminar una carrera de forma exitosa, véase Gráfico 3.

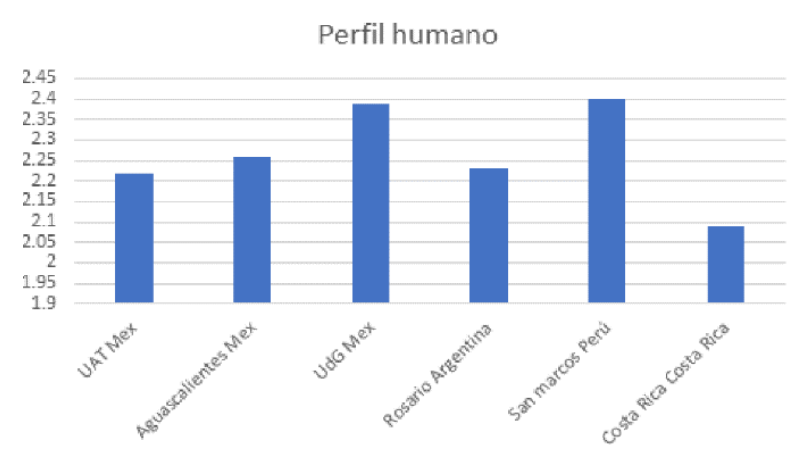

Gráfico 3 Representación conforme a su ítem y de acuerdo a la escala de Likert de la variable perfil humano del alumno en el rendimiento académico, en que los alumnos saben mantener la calma en situaciones estresantes, siendo la Universidad de Costa Rica, donde sus alumnos saben manejar situaciones de estrés, seguido por la Universidad Autónoma de Tamaulipas y Universidad Rosario, Argentina, después la Universidad de Aguascalientes, siendo las Universidades de Guadalajara y Universidad de San Marcos de Lima, Perú, donde existen situaciones de no saber manejar el estrés entre los jóvenes, siendo ciertas constantes en las seis universidades de Latinoamérica.

Fuente: Elaboración Propia

\section{Variable independiente, Rendimiento perfil escolar}

En esta variable dentro del rendimiento académico se contemplan 20 ítems.
Donde el primero, menciona que el estudiante se siente forzado a estudiar una licenciatura universitaria; el segundo, dice que me gustaría que existieran más actividades culturales; el tercero, menciona que me gustaría participar en actividades deportivas en la universidad; el cuarto, dice me gustaría participar en actividades que realiza mi escuela como conferencias; quinto, dice considero que dedico el tiempo necesario para las labores académicas; sexto, a veces siento necesidad de faltar a clases por motivos innecesarios; séptimo, me olvido fácilmente de cumplir los deberes académicos; octavo, suelo ser organizado para cumplir de forma eficiente los trabajos académicos; noveno, me gusta usar herramientas de aprendizaje; décimo, generalmente estudio un día antes de los exámenes; décimo primero, usualmente saco copias a los apuntes de mis compañeros de clase; décimo segundo, considero que me es fácil el aburrirme durante las clases; décimo tercero, me dificulta seguir adecuadamente las instrucciones del profesor; décimo cuarto, me es difícil poner atención en clases; décimo quinto, después de cierto tiempo se me es fácil perder la concentración; décimo sexto, se me dificulta trabajar en equipo; décimo séptimo, se me es fácil comprender los temas expuesto en el salón de clases; décimo octavo, usualmente se me olvidan los contenidos de las clases que ya he estudiado; décimo noveno, he pensado en la posibilidad de cambiarme de carrera; veinteavo, en el futuro me visualizo ejerciendo la carrera que estudio, véase Gráfico 4.

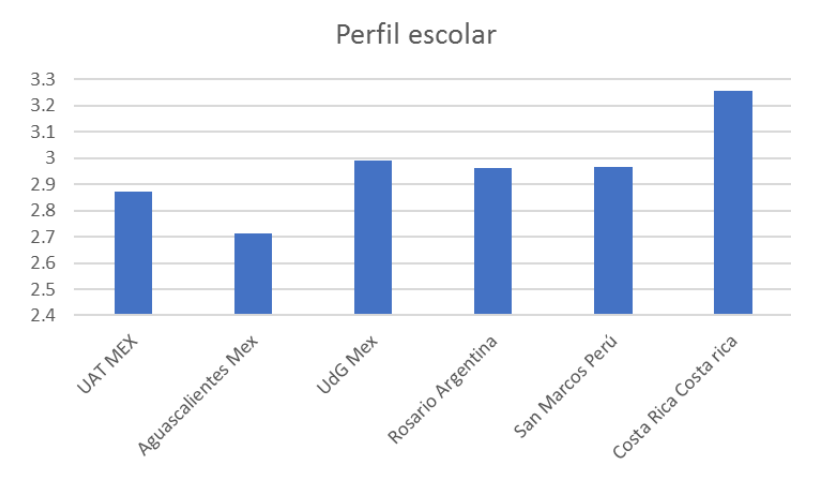

Gráfico 4 Representación conforme a su ítem y de acuerdo a la escala de Likert de la variable perfil escolar del rendimiento académico en donde se me olvida fácilmente cumplir los deberes académicos, siendo la Universidad de Aguascalientes donde sus alumnos tienden a olvidar las tareas o trabajos encargados por el profesor en el salón de clases seguido por la Universidad Autónoma de Tamaulipas y Universidad de Rosario, Argentina, posteriormente la Universidad de San Marcos, Lima, Perú, La Universidad de Guadalajara y la Universidad de Costas Rica es donde los alumnos no se les olvida realizar sus ejercicios o tareas que les encargan los docentes, siendo ciertas constantes en las seis universidades de Latinoamérica.

Fuente: Elaboración Propia 
En el Gráfico 5 la correlación cruzada de la variable del perfil humano con la variable del perfil escolar se ve sí hay una correlación cruzada del perfil escolar y donde se identifica como resultado que no hay una correlación cruzada y la Inter correlación en el desfase 1

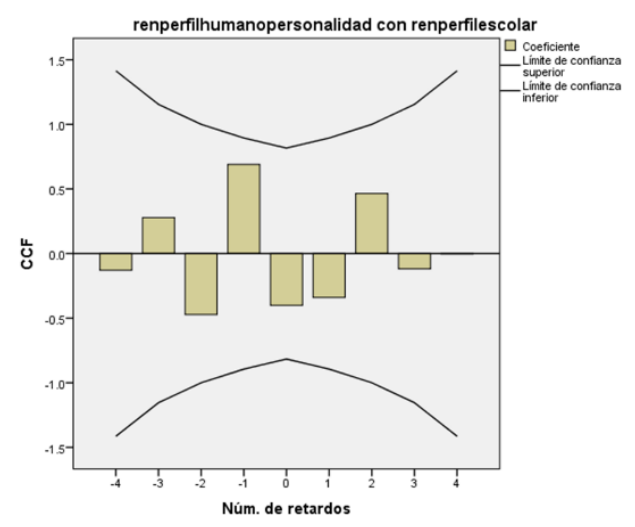

Gráfico 5 La Inter correlación de la existencia en dos variables, con el perfil humano y perfil escolar, donde no son significativas, ni su valor que no es absoluto en las medias iguales a cero y por las desviaciones estándar de la media, al no existir una auto correlación, donde el coeficiente tiende a dar negativos ante un límite de confianza hacia la baja con una muestra de 6 .

Fuente: Elaboración Propia

En el Gráfico 6 la correlación cruzada de la variable del perfil humano con la variable del perfil escolar se ve sí hay una correlación cruzada del perfil escolar y donde se identifica como resultado que no hay una correlación cruzada y la Inter correlación en el desfase 1.

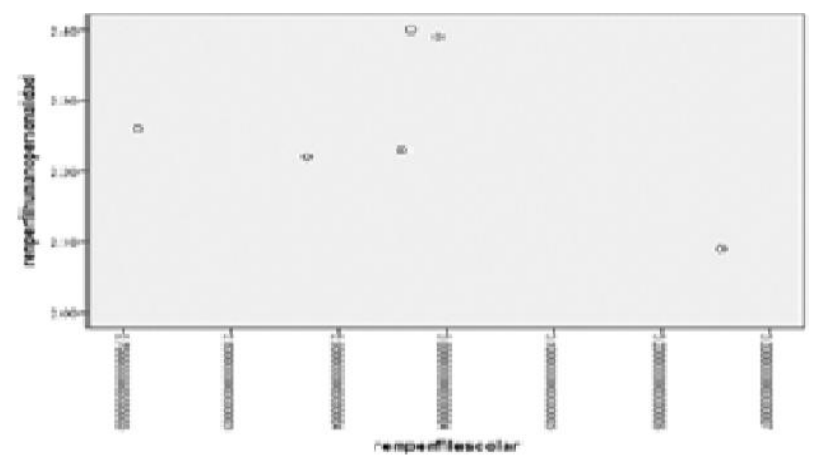

Gráfico $6 \mathrm{El}$ análisis multivariante es definido por Hair et al., (1999) que tiene el propósito medir y explicar y predecir el grado de relación de los valores teóricos, son combinaciones lineales de variables compuestas a partir de ponderaciones empíricas aplicadas y en este trabajo hacia al rendimiento académico y la ansiedad. Las matrices de correlaciones que se muestran, como se encuentran relacionadas las variables dependientes e independientes destacando puede existir una baja o alta correlación significativa con las variables como: perfil escolar, familias, ansiedad psíquica y ansiedad somática. Considerando la correlación de Pearson (r) es mayor que 0 por lo cual corresponderá a una correlación positiva con sentido directo y sí su cercanía al 0 es positiva pero baja. Fuente: Elaboración Propia
En la Tabla 1 se puede ver los datos estadísticos descriptivos de la investigación considerando la media y desviación típica de las variables consideradas y correlacionadas con pearson.

\begin{tabular}{|c|c|c|c|c|}
\hline \multicolumn{2}{|l|}{ Variables } & $\begin{array}{l}\text { Perfil } \\
\text { humano }\end{array}$ & $\begin{array}{l}\text { Perfil } \\
\text { escolar }\end{array}$ & $\begin{array}{l}\text { Ansiedad } \\
\text { somática }\end{array}$ \\
\hline $\begin{array}{l}\text { Perfil } \\
\text { humano }\end{array}$ & $\begin{array}{l}\text { Correlación } \\
\text { de Pearson } \\
\text { Sig } \\
\text { (bilateral) }\end{array}$ & 1 & $\begin{array}{r}-.401 \\
.431\end{array}$ & $\begin{array}{r}-.130 \\
.733\end{array}$ \\
\hline & $\mathrm{N}$ & 6 & 6 & 6 \\
\hline $\begin{array}{l}\text { Perfil } \\
\text { escolar }\end{array}$ & $\begin{array}{l}\text { Correlación } \\
\text { de Pearson } \\
\text { Sig } \\
\text { (bilateral) } \\
\end{array}$ & $\begin{array}{r}-.421 \\
.421\end{array}$ & 1 & .002 \\
\hline & $\mathrm{N}$ & 6 & 6 & 6 \\
\hline $\begin{array}{l}\text { Ansiedad } \\
\text { Somática }\end{array}$ & $\begin{array}{l}\text { Correlación } \\
\text { de Pearson } \\
\text { Sig } \\
\text { (bilateral) }\end{array}$ & $\begin{array}{r}-.150 \\
.733\end{array}$ & $\begin{array}{r}-.133 \\
.302\end{array}$ & 1 \\
\hline & $\mathrm{N}$ & 6 & 6 & 6 \\
\hline
\end{tabular}

Tabla 1 Variables de correlaciones. Donde se observan las variables con las correlaciones negativas y positivas de las variables perfil humano, perfil escolar y ansiedad somática. Notas. *La correlación es significativa en el nivel 0.05 (dos colas).** La correlación es significativa en el nivel 0.01 (dos colas), $\mathrm{N}=$ Número de participantes en el estudio.

Fuente: Elaboración Propia

\section{Correlacionas negativas}

La variable de ansiedad somática con perfil humano, las variables perfil escolar con perfil humano, perfil escolar y ansiedad somática.

En la Tabla 2 se puede ver la correlación entre dos variables, siendo ansiedad somática, la variable independiente perfil escolar, donde su correlación es negativa -.13, considerada como no significativa, ya que en ambas no se correlacionan en sentido inverso, considerando los datos sobre la muestra de las 6 universidades involucradas.

\begin{tabular}{|l|l|c|c|}
\hline & \multicolumn{3}{|c|}{$\begin{array}{l}\text { Ansiedad } \\
\text { somática }\end{array}$} \\
\hline $\begin{array}{l}\text { Ansiedad } \\
\text { somática }\end{array}$ & $\begin{array}{l}\text { Correlación } \\
\text { de Pearson } \\
\text { Sig (bilateral) }\end{array}$ & 1 & -.133 \\
\hline & $\mathrm{N}$ & 6 & .802 \\
\hline Perfil escolar & $\begin{array}{l}\text { Correlación } \\
\text { de Pearson } \\
\text { Sig (bilateral) }\end{array}$ & -.133 & 6 \\
\hline & $\mathrm{N}$ & 6 & 1 \\
\hline
\end{tabular}

Tabla 2 Correlación de variables de ansiedad somática y perfil escolar

Fuente: Elaboración Propia 


\section{Discusión}

Lo podemos decir, es que obtuvimos interesantes resultados al identificar la problemática sobre cada uno de los rendimientos académicos en cada país participante, considerando sus normas constitucionales, que seguramente ayudaron para identificar las estrategias utilizadas por cada gobierno para la educación que dedican a sus pueblos. Como se publica en Costa rica sobre el rendimiento académico del estudiantado universitario, constituye un factor imprescindible en el abordaje del tema de la calidad de la educación superior, debido a que es un indicador que permite una aproximación a la realidad educativa. (Díaz, Peio, Arias, Escudero, Rodríguez, Vidal, 2002). En materia de rendimiento académico en la educación superior, la mayoría de los estudios son cuantitativos, con un marcado interés en el campo económico y son pocas las investigaciones que hacen un abordaje cualitativo del problema y menos aplicando la neurocomputación biológica. por lo que en este estudio y de acuerdo al análisis y resultados obtenidos se propone que deben de considerarse nuevas estrategias o líneas de investigación donde incluyan a la neuroeducación dentro del desarrollo cognitivo docentes y estudiantil bajo el entendido de que la biología es el factor más importante de vida como son los telómeros y seguir realizando un estudio longitudinal de generaciones y cohortes precedentes, tanto del modelo cualitativo como cuantitativo, permitiendo así, que sean realmente significativas sus variables de forma con estudios transversales a corto, mediano y largo plazo, partiendo de sus posturas dependientes e independientes, según los hallazgos que en la educación superior se van desarrollando.

Finalmente, este trabajo puede ser la base de un análisis más detallado por las características donde el rendimiento académico es la suma de diferentes y complejos factores o psicosociales que actúan en la persona que aprende, y ha sido definido con un valor atribuido al logro del estudiante en las tareas académicas. Se mide mediante las calificaciones obtenidas, mas no con el sentido de ver el tipo de ansiedad psíquica o somática y con una valoración cuantitativa, cuyos resultados se muestran en las materias ganadas o perdidas, la deserción y el grado de éxito académico Pérez, Ramón, Sánchez (2000), Vélez van, Roa (2005). se pudieron obtener, del rendimiento académico y la ansiedad (analizada con detalle).
Existe la posibilidad de implementar nuevos tipos de análisis más rigurosos a intervalos específicos de los promedios escolares como: resultados de las evaluaciones en las asignaturas cursadas, la impartición de nuevas asignaturas por docentes recién contratados, un análisis de tipo multivariado (ANOVA y multi ANOVA), el trabajo de investigación permitirá abrir nuevas líneas de investigación para ir complementando la neurocomputación biológica, la cual servirá como la siguiente fase del próximo proyecto de investigación sobre la neurocomputación biológica dedicada a la interpretación de las instrucciones que se manejan en los exámenes.

\section{Agradecimientos}

A las universidades latinoamericanas como: Universidad Nacional de Rosario, Argentina; Universidad Nacional Mayor de San Marcos de Lima, Perú; Universidad de Costa Rica, Costa Rica; Benemérita Universidad de Guadalajara, Universidad Autónoma de Aguascalientes y Universidad Autónoma de Tamaulipas, México.

\section{Conclusiones}

Los resultados del análisis nos permitieron extraer una base de datos, para tener buenas conclusiones sobre el trabajo de investigación que se planteó bajo el seguimiento de una tesis doctoral con el título sobre los factores psicosociales en el rendimiento académico de la Universidad Autónoma de Tamaulipas y permitió contrastar sus variables con escenarios locales, al manejo internacional con los países latinoamericanos, permitiendo así poder visualizar con mayor representatividad el manejo de los datos obtenidos a través de los instrumentos empleados en la investigación y la información enfocada al rendimiento académico.

En cuanto a los objetivos; primero, si el rendimiento académico y las habilidades psicosociales bajo la identidad latinoamericana, en este concepto universitario los paises como argentinos, peruanos, costarricenses y mexicanos, se incremente su nivel académico para sus alumnos y considerando la mejora en el desarrollo del estudio académico; segundo, analizar que si el aprovechamiento académico considera a la neurocomputación biológica, permitiendo identificar que existe cierto grado de ansiedad somática en los estudiantes, cuando tienen actividades escolares y académicas.

JIMÉNEZ-GALÁN, Joel Luis, FALCONE-TREVIÑO, Giuseppe Francisco, TINAJERO-MALLOZZI, Zaida Leticia y SERNAHINOJOSA, José Antonio. Rendimiento académico universitario en países latino americanos bajo la neurocomputación biológica. Revista de Gestión Universitaria. 2019 
Tercero, al medir los distintos indicadores entre las habilidades sociales y los promedios académicos entre los alumnos se pudo identificar que el rendimiento académico, factores y habilidades psicosociales, hay diferencias marcadas al trabajo en equipo y aspectos importantes que se deberán de tomar en cuenta en las universidades públicas de los países participantes como México, Universidad Autónoma de Aguascalientes, Benemérita Universidad de Guadalajara, Universidad Autónoma de Tamaulipas; Argentina, Universidad Nacional de Rosario; Perú, Universidad Nacional Mayor de San Marcos de Lima; Costa Rica, Universidad de Costa Rica. Considerando como un referente indicativo muy importante que es el perfil escolar y el perfil humano.

Porque se identificó que el rendimiento académico es un problema enorme cuando no se toman en cuenta estos aspectos, problemas que se deben de atacar con estrategias innovadoras y modelos metodológicos de la cuarta generación, sobre la enseñanza y aprendizaje, donde se demuestre desde la contratación de los docentes, hasta la definición congruente del perfil de un egresado, en cualquier programa académico, considerando así utilizar nuevas variables para futuras investigaciones como: el ambiente escolar, familias, la ansiedad psíquica, el promedio y la alimentación del estudiante, etc., con la idea de identificar soluciones para las nuevas generaciones sustentadas en mejores valores e indicadores en las universidades públicas de los países participantes.

Ya que se dice que hay escritos sobre la educación en diferentes países como por ejemplo; en México, "será de calidad, con base en el mejoramiento constante y el máximo logro académico de los educandos"; Perú, "el educando tiene derecho a una formación que respete su identidad, así como al buen trato psicológico y físico"; Costa Rica, "la educación pública será organizada como un proceso integral correlacionado en sus diversos ciclos"; Argentina, "desarrollar armónicamente, todas las facultades del ser humano y fomentará en él, a la vez, el amor a la Patria, el respeto a los derechos humanos y la conciencia de la solidaridad internacional".
También considerando el poder de determinar las frecuencias, correlaciones, regresiones, desviaciones típicas, coeficientes, especificaciones Bootstrap y datos estadísticos descriptivos para identificar los siguientes puntos en cuanto a las variables independientes y sus indicadores en las medias sobre el perfil humano, la responsabilidad académica, y ansiedad somática, se consideraron que las mejoras, fueron correlaciones positivas, con una efectividad superior al $99 \%$.

El realizar un análisis exhaustivo de las matrices de correlación cruzada que brindan información útil en el funcionamiento del rendimiento académico. Las observaciones que se realizaron fueron para diferentes retardos de tiempo en la matriz de correlación del coeficiente de variación de Spearman por la interdependencia entre las variables. En lo que se refiere a la $\mathrm{p}$. no se puede realizar una correlación puntual, con su respectiva contraparte, debido a que no posee información útil que pueda servir para describir su comportamiento por su respectivo orden. En la tabla 3 se muestran las posiciones que obtuvieron las universidades participantes conforme a los resultados estadísticos obtenidos por rendimiento académico.

La Tabla 3 muestra las universidades que demostraron tener más bajo rendimiento académico y mayor ansiedad (1), hasta la universidad con mejor rendimiento académico y menor ansiedad (6).

\begin{tabular}{|r|l|}
\multicolumn{1}{|c|}{ Número } & \multicolumn{1}{c|}{ Universidad } \\
\hline 1 & Autónoma de Aguascalientes. México \\
\hline 2 & Nacional Mayor de San Marcos Lima, Perú \\
\hline 3 & Autónoma de Tamaulipas, México \\
\hline 4 & Benemérita de Guadalajara, México \\
\hline 5 & Nacional de Rosario, Argentina \\
\hline 6 & De Costa Rica, Costa Rica \\
\hline
\end{tabular}

Tabla 3 Listado de universidades según su rendimiento académico de menor a mayor

\section{Referencias}

Abarca, A., \& Sánchez, M. (2005). La deserción estudiantil en la educación superior: El caso de la Universidad de Costa Rica. Actualidades investigativas en educación. Recuperado de: http://revista.inie.ucr.ac.cr/ uploads/ tx_magazine/ deserc_01.pdf

Acevedo, J. (2009). Del problema a la presentación de proyectos de investigación. México: Valle de Candamo.

JIMÉNEZ-GALÁN, Joel Luis, FALCONE-TREVIÑO, Giuseppe Francisco, TINAJERO-MALLOZZI, Zaida Leticia y SERNAHINOJOSA, José Antonio. Rendimiento académico universitario en países latino americanos bajo la neurocomputación biológica. Revista de Gestión Universitaria. 2019 
Anchondo, S., \& de Haro, M. (2016). El mestizaje es un mito, la identidad cultural sí importa. Miscegenation is a myth, cultural identity does matter. México: Istmo. Consultado el 24 de agosto de 2017.

Guadagni, A. (2015). Clarín.com. Sociedad, Educación. Buenos Aires, Argentina.

Bernales, E. (1999). La Constitución de 1993. Lima: Ediciones Constitución y Sociedad, 3.

Bilbao, R., \& Rivas, A. (2011). Las provincias y las TIC: Avances y dilemas de política educativa. Buenos Aires: CIPPEC.

Biotic (2014). Área de informática y salud pública. Instituto Carlos III-Proyecto Genoma Humano. Madrid. España. Recuperado de: http://infobio chip.isciii.es

Bravo, Z., Katherine, L., Guillen, F., Elda, D., Nuñez, D., Froilan, E. Arias, I., Gallardo, M. \& Pilar, C. (2019). La imaginación creativa de estudiantes universitarios de la especialidad de Psicología. Apuntes Universitarios, 9(1), 68-84. Recuperado de: https://dialnet.unirioja.es/servlet/ articulo? codigo $=6816598$

Caetano, G. (2015). ¿Hacia un nuevo paradigma integracionista en el MERCOSUR? Contextos y desafíos de la encrucijada actual. Relaciones Internacionales, $\mathrm{N}^{\circ} 30$, octubre.

Campos, R., Barrantes, R., \& Raventós, H. (2016). Cuatro grupos étnicos componen genética de los costarricenses. Seminario Universidad de Costa Rica.

Carrión, E. (2002). Validación de características al ingreso como predictores del rendimiento académico en la carrera de medicina. Educación Médica Superior, v.16, n.1. Ciudad de la Habana, ene. - mar. Recuperado de http://scielo.sld.cu/ script=sci_arttext scielo.php? 21412002000100001

Cavero, M. (2019). Inteligencia emocional y rendimiento académico en alumnos de educación secundaria obligatoria.Revista Española de Orientación y Psicopedagogia, 30(1), 75-92. Recuperado de: http://revistas.uned.es/index.php/ reop/article/view/25195
Chóliz, M. (2005). Psicología de la emoción: El proceso emocional. Recuperado de: http://www.uv.

es/=choliz/Proceso\%20emocional.pdf

CONAPO. (2011). Índices de Marginación 2010. México, Distrito Federal: Edición digital. Recuperado de: http://www.conapo.gob.mx

CONAPO. (2012). Extracto de proyecciones de población 2012-2050. (p.3). México: Consejo Nacional de Población.

Corvalán, F. (2013). 50 años de representaciones sociales y psicología: Campo psy, bifurcaciones $y$ desafios. Estudios Contemporáneos da Subjetividad, 3(1), 115-127. Recuperado de: http://www. periodicoshumanas.uff.br/ecos/article/viewFile/ $1055 / 823$

El comercio. (2016). Perú es el país con bajo rendimiento escolar de Sudamérica. Perú.

Erazo, O. (2012). El rendimiento académico, un fenómeno de múltiples relaciones $y$ complejidades. Revista Vanguardia Psicológica Clínica Teórica y Práctica. Universidad Manuela Beltrán. Volumen 2, Número 2, Bogotá D.C. Colombia octubre - Marzo. Recuperado de: https://dialnet.unirioja.es/servlet/ articulo? codigo $=4815141$

Ferreyra, H., Cingolani, M., Eberle, M., Gallo, G., Larrovere, C., Luque, M., Pasut, M., Peretti, G., \& Rimondino, R. (2009). Educación Secundaria Argentina. Propuestas para superar el diagnóstico y avanzar hacia su transformación. Buenos Aires: Ediciones Novedades Educativas y Academia Nacional de Educación.

Gómez, V., Rosales, S., Marín, G., García, J., \& Guzmán, J. (2012). Correlación entre el examen de selección y el rendimiento académico al término de la carrera de Medicina. Revista de Educación Médica Superior, vol. 26, no. 4. Ciudad de la Habana, oct. - dic. Recuperado de: http://scielo.sld.cu/scielo.php?script=sci_abstra ct $\quad$ \&pid $=$ S0864-21412012000400003\& $\operatorname{lng}=\mathrm{es} \& \mathrm{nrm}=\mathrm{iso}$

Kessler, G. (2004). Sociología del delito amateur. Buenos Aires: Paidós.

Hair, J. et al. (1999). Analisis Multivariante. Madrid: Prentice Hall.

JIMÉNEZ-GALÁN, Joel Luis, FALCONE-TREVIÑO, Giuseppe Francisco, TINAJERO-MALLOZZI, Zaida Leticia y SERNAHINOJOSA, José Antonio. Rendimiento académico universitario en países latino americanos bajo la neurocomputación biológica. Revista de Gestión Universitaria. 2019 
Hamilton, M. (1969). Diagnosis and rating of anxiety. In Studies of Anxiety, Lander, MH. Brit J Psychiat Spec Pub; 3:76-79.

Helsinki. (2017). Principios éticos para investigación médica en seres humanos. (C) World Medical Association, Inc.

Huamaní, C., Gutiérrez, C., \& Mezones, E. (2011). Correlación y concordancia entre el examen nacional de medicina y el promedio ponderado universitario: análisis de la experiencia peruana en el periodo 2007 - 2009. Revista Peruana de Medicina Experimental y Salud Publica, vol. 28, no. 1. Lima. Mar. 2011. Recuperado de: http://www.scielo.org.pe/scielo.php?pid=S1726 -46342011000100010 \&script=sci_arttext

INFOBASE. (2018). Cada 8 minutos un alumno argentino repite o abandona la secundaria.

Jiménez, D. (2013). Beneficios de la Certificación y Acreditación en la Educación. Revista COEPES, Año 3, No. 8. Obtenido el 13 de marzo de 2015. Recuperado de: http://www.revistacoepesgto.mx

/revistacoepes8/beneficios-de-la-certificacion$\mathrm{y}$-acreditacion-en-la-educacion

Jiménez, J. (2011). Análisis de factores psicosociales en el rendimiento académico: UAMCEH y UAMCAV, Cd. Victoria, Tamaulipas. México.

Ley Nacional de Educación. (2007). Ley $N^{\circ}$. 26.206. Hacia una educación de calidad para una sociedad más justa. Buenos Aires: Ministerio de Educación Ciencia y Tecnología.

López, M., Fernández, E., Amezcua, J., \& Pichardo, M. (2000). ¿Difieren en autoconcepto los adolescentes con baja visión de los adolescentes con visión normal? Integración, 33, 14-20.

Lucas, S. (2001). Effectively Maintained Inequality: Education transitions, track mobility and social background effects. American Journal of Sociology, vol. 106, núm. 6, pp. 1642-1690.

Margulis, M., \& Urresti, M. (1998). La segregación negada: Cultura y discriminación social. Buenos Aires: Biblos.
Martínez, E., Fonseca, R., \& Tapia, H. (2019). Implementación de Rediseños Curriculares Universitarios en Educación, una Tarea Compleja. Formación universitaria, 12(3), 55$66 . \quad$ Recuperado de: https://scielo.conicyt.cl/scielo.php? script=sci_ arttext\&pid=S0718-50062019000300055

MEDMAR. (2008). Evaluación integral del rendimiento escolar en educación superior 2008. Un instrumento de evaluación.

Páez, A., Gaytán, N. \& Sánchez, D. (2019). Construcción e implementación de un modelo para predecir el rendimiento académico de estudiantes universitarios mediante el algoritmo Nä̈ve Bayes. Diálogos sobre educación, (19). Recuperado de: http://dialogossobreeducacion.cucsh.udg.mx/in dex.php/DSE/article/view/509

Padua, L. (2019). Factores individuales y familiares asociados al bajo rendimiento académico en estudiantes universitarios. Revista mexicana de investigación educativa, 24(80), 173-195. Recuperado de: https://www.comie.org.mx/revista/ v2018/rmie/index.php/nrmie/article/view/1242

Ramírez, I. (2009). La palabra de la reforma en la república de las letras. Una antología general. Selección y estudio preliminar de Liliana Weinberg. México: FCE/Fundación para las Letras Mexicanas/UNAM.

Roselli, N. (2011). Teoría del aprendizaje colaborativo y teoría de la representación social:Convergencias y posibles articulaciones. Revista Colombiana de Ciencias Sociales, 2(2), 173-191. Recuperado de: https://doi.org/ 10.21501/ issn.221 6-1201

Sandoval, J., \& Rodríguez, F. (2013). Historia Genética das Populações peruanas. Tesis doctoral en genética aplicada. (Sandoval, J.). Universidad Federal de Minas Gerais. Hospedador científico: Research Gat.

Tomatis, M., Burrone, M., Romero, D., Novella, M., Olivero, M., Antuña, A., Lucchese, M., Enders, J., \& Fernández, A. (2016). Validez predictiva del examen de ingreso a la carrera de medicina de la Facultad de Ciencias Médicas $(U N C)$. Revista de educación, año 7, no. 9, pp. 357 - 368. Recuperado de: http://fh.mdp.edu.ar/revis tas/index.php/r_educ/ article/view/1917/1855

JIMÉNEZ-GALÁN, Joel Luis, FALCONE-TREVIÑO, Giuseppe Francisco, TINAJERO-MALLOZZI, Zaida Leticia y SERNAHINOJOSA, José Antonio. Rendimiento académico universitario en países latino americanos bajo la neurocomputación biológica. Revista de Gestión Universitaria. 2019 
Vélez, A., \& Roa, C. (2005). Factores asociados al rendimiento académico en estudiantes de medicina. Revista de Educación Médica, vol. 8, no. 2, jun. Recuperado de: http://www.scielo.org.mx/ scielo.php?script=sci_arttext\&pid=S018533252011000400002

Zabalza, M. (2003). Competencias docentes del profesorado universitario: Calidad y desarrollo profesional. Madrid: Narcea Ediciones.

\section{Anexo}

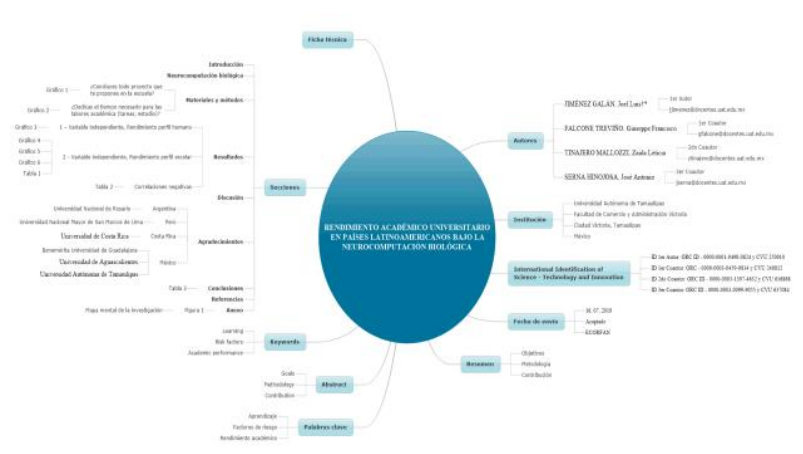

Figura 1 Mapa mental de la investigación

Fuente: Elaboración Propia 